\title{
Risk factors for recurrent Clostridium difficile infection in hematopoietic stem cell transplant recipients
}

\author{
A.M. Huang, B.L. Marini, D. Frame, D.M. Aronoff, J.L. Nagel. Risk \\ factors for recurrent Clostridium difficile infection in hematopoietic \\ stem cell transplant recipients. \\ Transpl Infect Dis 2014: 16: 744-750. All rights reserved
}

Abstract: Background. Recurrent Clostridium difficile infection (CDI) represents a significant burden on the healthcare system and is associated with poor outcomes in hematopoietic stem cell transplant (HSCT) patients. Data are limited evaluating recurrence rates and risk factors for recurrence in HSCT patients.

Methods. HSCT patients who developed CDI between January 2010 and December 2012 were divided into 2 groups: non-recurrent CDI (nrCDI) and recurrent CDI (rCDI). Risk factors for rCDI were compared between groups. Rate of recurrence in HSCT patients was compared to that in other hospitalized patients.

Results. CDI was diagnosed in 95 of $711 \mathrm{HSCT}$ patients $(22 \mathrm{rCDI}$ and $73 \mathrm{nrCDI}$ ). Recurrence rates were similar in HSCT patients compared with other hospitalized patients $(23.2 \%$ vs. $22.9 \%$, $P>0.99$ ). Patients in the rCDI group developed the index case of CDI significantly earlier than the nrCDI group (3.5 days vs. 7.0 days after transplant, $P=0.05$ ). On univariate analysis, patients with $\mathrm{rCDI}$ were more likely to have prior history of CDI and neutropenia at the time of the index CDI case. Neutropenia at the time of the index CDI case was the only independent predictor of rCDI (78.8 vs. $34.8 \%$, $P=0.006)$ on multivariate analysis.

Conclusions. The rate of rCDI was similar between HSCT and other hospitalized patients, and the majority of patients developed the index case of CDI within a week of transplantation. Neutropenia at the index CDI case may be associated with increased rates of rCDI.

\author{
A.M. Huang', B.L. Marini', D. Frame', \\ D.M. Aronoff', J.L. Nagel' \\ 'Department of Pharmacy Services and Clinical Sciences, \\ University of Michigan Health System and College of \\ Pharmacy, Ann Arbor, Michigan, USA, ${ }^{2}$ Division of \\ Infectious Diseases, Department of Internal Medicine, \\ University of Michigan Health System and Medical \\ School, Ann Arbor, Michigan, USA
}

Key words: recurrent Clostridium difficile; stem cell transplant; recurrence

\author{
Correspondence to: \\ Jerod Nagel, PharmD, BCPS (AOID), Department of \\ Pharmacy Services and Clinical Sciences, University \\ of Michigan Health System and College of \\ Pharmacy, 1111 E. Catherine St., Room 300, \\ Ann Arbor, Ml 48109, USA \\ Tel: 7349362264 \\ Fax: 7349367027 \\ E-mail: nagelj|@med.umich.edu \\ (or) \\ Angela Huang, PharmD, BCPS, Froedtert \& The \\ Medical College of Wisconsin, 9200 W. Wisconsin \\ Ave., Milwaukee, WI 53202, USA \\ Tel: 4148059291 \\ Fax: 4148056702 \\ E-mail: angela.huang@froedtert.com
}

Received 29 January 2014, revised 31 March 2014, accepted for publication 4 May 2014

DOI: 10.1111/tid.12267

Transpl Infect Dis 2014: 16: 744-750
Clostridium difficile infection (CDI) is the leading cause of nosocomial infectious diarrhea in the United States. The incidence of CDI in patients undergoing hematopoietic stem cell transplantation (HSCT) is approximately $5-30 \%$ compared with $1-2 \%$ in the general hospitalized population (1-4). HSCT patients are commonly exposed to risk factors for the development of CDI, including prolonged length of hospitalization, exposure to broad-spectrum antibiotics, receipt of acid-suppression therapy, disrupted integrity of intestinal mucosa, and profound immunosuppression (5).
Despite higher prevalence of CDI in HSCT recipients, limited information is available evaluating recurrent infection. In addition, risk factors for recurrent infection in HSCT have not been thoroughly evaluated. Therefore, the objective of this study was to determine the incidence of recurrent CDI (rCDI) in HSCT patients compared with that in the general hospitalized population, and to examine risk factors for rCDI. Characterization of risk factors for rCDI may aid in identification of patient populations that might benefit from prevention strategies or targeted initial treatment. 


\section{Patients and methods}

This retrospective case-control study was conducted at the University of Michigan Health System (UMHS) and received investigational review board approval. Patients were included if they underwent HSCT at UMHS between January 2010 and December 2012 and developed CDI 7 days before HSCT through 1 year post transplant. Patients were considered to have CDI if they exhibited symptoms consistent with CDI and had a stool sample test positive for $C$. difficile in the UMHS Clinical Microbiology Laboratory. Stool testing was performed using the C. DIFF QUIK CHEK COMPLETE $^{\circledR}$ test (Alere/TECHLAB ${ }^{\circledR}$, Inc.; Blacksburg, Virginia, USA) for $C$. difficile glutamate dehydrogenase antigen and toxins A or B by enzyme immunoassay. All glutamate dehydrogenase-positive/toxin-negative stool tests were subjected to analysis for the $t c d B$ gene by real-time polymerase chain-reaction assay (BD Gene$\mathrm{Ohm}^{\mathrm{TM}}$ Cdiff Assay; Franklin Lakes, New Jersey, USA). Pediatric patients $<2$ years of age were excluded. For patients who received $>1$ HSCT in a calendar year, only the first transplant was evaluated.

The study population was divided into 2 groups for analysis: rCDI and non-recurrent CDI (nrCDI); rCDI was defined as a repeat positive CDI occurring within 6 months after the end of treatment for the index case (first episode of CDI after transplantation). To compare rates of rCDI in general hospitalized population with those in HSCT patients, incidence of rCDI was also estimated for all hospitalized patients between January 2010 and December 2012. Patients who received an HSCT in the year before diagnosis of the index episode of CDI were excluded from this group; thus no patients were included in both groups.

Data collection points included patient demographics, CDI severity and complications, CDI treatment, traditional CDI risk factors for recurrence, potential HSCT-specific risk factors for recurrence, and timing and frequency of rCDI. Traditional risk factors for recurrence include age $>65$ years, broad-spectrum antibiotics, acid-suppression therapy, history of CDI, and hospitalization $\geq 14$ days. History of CDI was defined as CDI in the year before transplantation and occurring before the index episode. Additional HSCTrelated factors were also collected and analyzed as potential predictors for recurrent disease including type of transplant (autologous vs. allogeneic [autoHSCT vs. alloHSCT]), alloHSCT from a matched related or unrelated donor, myeloablative conditioning regimen, neutropenia at the time of the index case (defined as an absolute neutrophil count $<500$ cells $/ \mathrm{mm}^{3}$ ), the index CDI case occurring during hospitalization for transplantation, use of prophylactic immunosuppressive medications, use of immunosuppression for treatment of graft-versus-host disease (GVHD), diagnosis of biopsy-proven cytomegalovirus colitis, and diagnosis of GVHD. All risk factors were collected from the day of positive CDI result to the end of the CDI treatment course. All patients transplanted at UMHS were followed by our transplant team for both inpatient and outpatient care.

Severe CDI was defined as an episode of CDI resulting in need for intensive care unit admission, interventional surgery, or death within 30 days of CDI diagnosis (6). GVHD grading according to Glucksberg criteria (7) was captured for each alloHSCT patient and recorded in a transplant database. GVHD was further categorized as acute (onset $\leq 100$ days post transplant) or chronic (onset $>100$ days post transplant). Patients who received tumor necrosis factor-alpha inhibitors or rituximab for the treatment of GVHD within 90 days before CDI onset were considered to be on immunosuppression because of the prolonged immunosuppressive effect of these agents.

\section{Statistical analysis}

All statistical analyses were performed using SPSS Software Version 20.0 (SPSS, Inc., Chicago, Illinois, USA). Demographic data were analyzed by descriptive statistics, categorical data were analyzed by 2-tailed Student $t$-test, and dichotomous data were analyzed by Pearson chi-square test. Mann-Whitney $U$-tests were performed for non-normally distributed factors.

Unconditional logistic regression analysis was performed to evaluate factors associated with $\mathrm{rCDI}$ at the time of the index CDI, and included traditional and HSCT-related factors. Factors with $P$-value $\leq 0.2$ on the bivariate model were included in a multivariate unconditional logistic regression analysis. In the final model, a $P$-value $\leq 0.05$ (2-tailed) was considered statistically significant. An additional bivariate sub-analysis, evaluating alloHSCT and autoHSCT recipients, was performed to determine if risk factors for recurrence differ between these 2 populations. Several variables, including immunosuppression, GVHD, and treatment of GVHD, were not applicable to autoHSCT patients and were therefore not included in the analysis for that population. No multivariable regression analysis was performed in the subgroups owing to small sample sizes. 


\section{Results}

CDI was diagnosed in 95 of 711 HSCT patients (13.4\%) from January 2010 to December 2012, which included 330 alloHSCTs and 381 autoHSCTs. The overall incidence of CDI was $18.2 \%$ (60 of 330 patients) in alloHSCT recipients and 9.2\% (35 of 381 patients) in autoHSCT recipients, $P<0.001$ (Fig. 1). CDI rate in the HSCT population during the study period was approximately 1.75 per 10,000 patient days. The average age of the whole cohort was 45 years and 53.7\% were male.

RCDI occurred in 22 patients, and the incidence of rCDI did not differ between alloHSCT and autoHSCT recipients $(23.3 \%$ vs. $22.9 \%, P>0.99)$. When compared with all inpatients during the same time period, the recurrence rate was not significantly higher in HSCT patients $(18.5 \%$ vs. $23.2 \%, P=0.283)$. In the rCDI group, 16 patients had 1 episode of recurrence (72.7\%), 4 patients experienced 2 recurrences (18.2\%), and 2 patients had 3 recurrences (9.1\%). Among rCDI patients, $31.8 \%$ developed recurrence $<4$ weeks after the end of treatment for their index case, $45.5 \%$ at 4 12 weeks, and $22.7 \%$ at $>12$ weeks (Fig. 2).

Age, gender, and incidence of severe CDI did not significantly differ between the rCDI and nrCDI groups (Table 1). Overall, the majority of patients developed their index case of CDI during day -7 to day +14 post transplant (Fig. 3). Patients in the rCDI group developed their index case of CDI significantly earlier than patients in the nrCDI group (3.5 days vs. 7.0 days, $P=0.05$ ). All patients in the rCDI group and $95.9 \%$ of patients in the nrCDI group received oral metronidazole for at least part of their CDI treatment, and a proportion were switched to oral vancomycin or initiated on combination therapy at some time during their

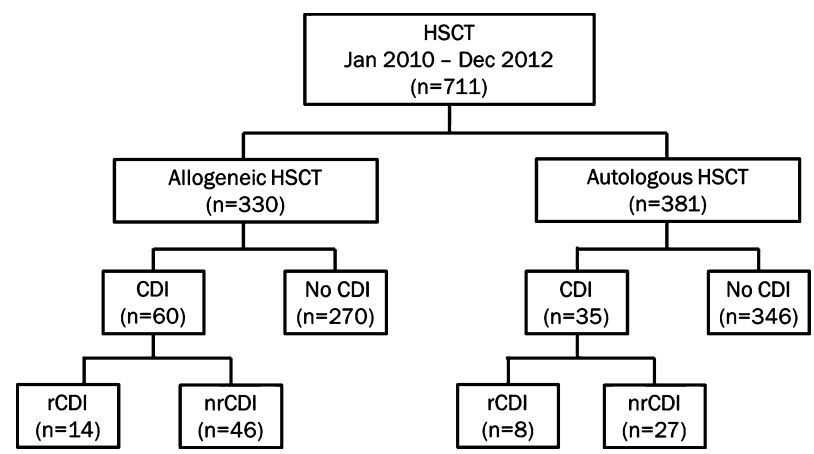

Fig. 1. Study population. HSCTs, hematopoietic stem cell transplants; CDI, Clostridium difficile infection; r, recurrent; nr, nonrecurrent.

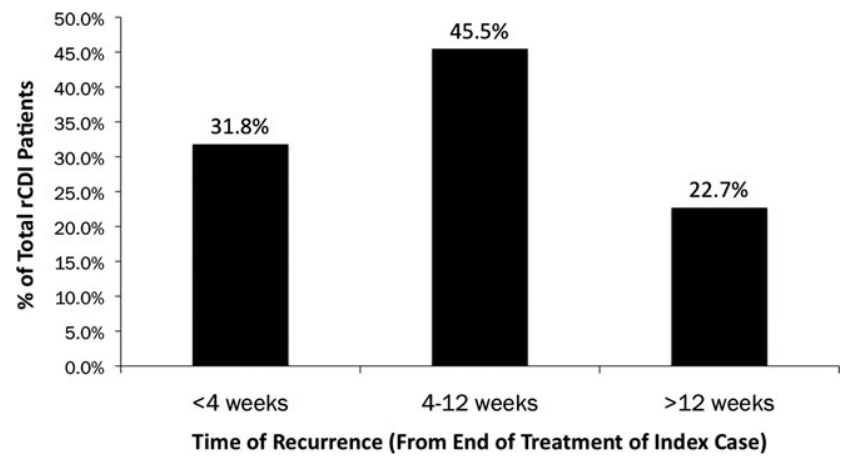

Fig. 2. Timing of recurrence in recurrent Clostridium difficile infection (rCDI) patients.

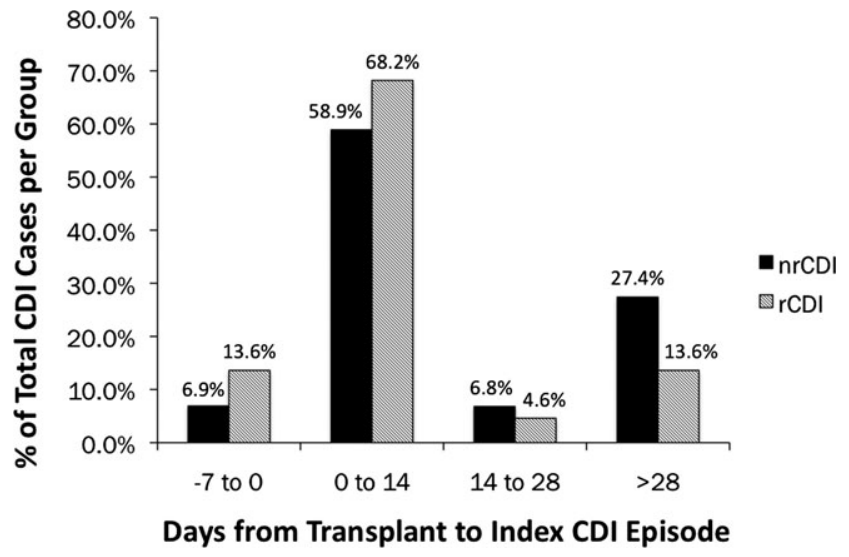

Fig. 3. Timing of initial Clostridium difficile infection (CDI) episode with relation to transplant date; nr, non-recurrent; r, recurrent.

treatment course. One patient in the nrCDI group received vancomycin rectal enemas. No significant differences were seen between the 2 groups with regard to $\mathrm{CDI}$ antimicrobial treatment choice or duration of therapy (Table 2).

Traditional risk factors for recurrence and HSCTrelated factors were compared between the rCDI and nrCDI groups via bivariate analysis. Among traditional risk factors for recurrence, history of CDI in the year before transplantation was seen frequently in the rCDI population $(22.7 \%$ vs. $6.8 \%, P=0.048)$, but acid-suppression therapy, broad-spectrum antibiotic use, and hospital stay $\geq 14$ days were not significantly different between the 2 groups. Analysis of HSCT-related factors revealed significantly more patients in the rCDI group were neutropenic during their index case of CDI (59.1\% vs. $28.8 \%, P=0.012)$. No other HSCT-related factors were statistically significantly different between the rCDI and nrCDI groups.

Neutropenia at the time of the index case was found to be the only independent predictor of recurrent 


\begin{tabular}{|c|c|c|c|}
\hline & $\begin{array}{l}\text { Recurrent (rCDI) } \\
(n=22)\end{array}$ & Non-recurrent $(\mathrm{nrCDI})(n=73)$ & $P$-value \\
\hline \multicolumn{4}{|l|}{ Demographics } \\
\hline Age at index case (mean $\pm \mathrm{SD}$ ) & $41.46 \pm 20.9$ & $45.5 \pm 21.3$ & 0.460 \\
\hline Male & $11(50.0)$ & $40(54.8)$ & 0.808 \\
\hline Severe CDI & $3(13.6)$ & $5(6.8)$ & 0.820 \\
\hline \multicolumn{4}{|l|}{ Traditional risk factors for recurrence } \\
\hline Age $>65$ & $1(4.6)$ & $7(9.6)$ & 0.677 \\
\hline History of CDI & $5(22.7)$ & $5(6.8)$ & 0.048 \\
\hline Hospitalization $\geq 14$ days & $22(100.0)$ & $70(95.9)$ & $>0.99$ \\
\hline \multicolumn{4}{|l|}{ Acid-suppression therapy } \\
\hline PPI & $9(40.9)$ & $44(60.3)$ & 0.143 \\
\hline H2RA & $9(40.9)$ & $19(26.0)$ & 0.193 \\
\hline \multicolumn{4}{|l|}{ Broad-spectrum antibiotics } \\
\hline Carbapenems & $2(9.1)$ & $1(1.4)$ & 0.133 \\
\hline Cephalosporins & $14(63.6)$ & $32(43.8)$ & 0.144 \\
\hline Clindamycin & $1(4.6)$ & $0(0.0)$ & 0.232 \\
\hline Fluoroquinolones & $11(50.0)$ & $24(32.9)$ & 0.207 \\
\hline $\mathrm{BL} / \mathrm{BLIS}$ & $3(13.6)$ & $19(26.0)$ & 0.266 \\
\hline Other ${ }^{1}$ & $7(31.8)$ & $16(21.9)$ & 0.398 \\
\hline \multicolumn{4}{|l|}{ HSCT-related factors } \\
\hline Index case during hospitalization for HSCT & $18(81.8)$ & $48(65.8)$ & 0.192 \\
\hline Neutropenic $($ ANC $<500$ ) at index case & $13(59.1)$ & $21(28.8)$ & 0.012 \\
\hline \multicolumn{4}{|l|}{ Type of transplant } \\
\hline Allogeneic & $14(63.6)$ & $46(63.0)$ & $>0.99$ \\
\hline Matched (\% of allogeneic HSCTs) & $12(85.7)$ & $41(89.1)$ & 0.661 \\
\hline \multicolumn{4}{|l|}{ Conditioning regimen } \\
\hline Myeloablative & $19(86.4)$ & $58(79.5)$ & 0.552 \\
\hline Immunosuppression (prophylaxis) & $13(59.1)$ & $43(58.9)$ & \\
\hline Tacrolimus & $12(54.5)$ & $40(54.8)$ & $>0.99$ \\
\hline Mycophenolate & $6(27.3)$ & $16(21.9)$ & 0.578 \\
\hline Cyclosporine & $0(0.0)$ & $2(2.7)$ & $>0.99$ \\
\hline Methotrexate & $5(22.7)$ & $22(30.1)$ & 0.597 \\
\hline Other $^{2}$ & $7(31.8)$ & $23(31.57)$ & $>0.99$ \\
\hline \multicolumn{4}{|l|}{ Immunosuppression (treatment for GVHD) } \\
\hline High-dose steroids ( $>0.5 \mathrm{mg} / \mathrm{kg}$ prednisone equivalent) & $1(4.5)$ & $10(13.7)$ & 0.448 \\
\hline TNF-alpha inhibitor & $0(0.0)$ & $2(2.7)$ & $>0.99$ \\
\hline Rituximab & $0(0.0)$ & $1(1.4)$ & $>0.99$ \\
\hline Other ${ }^{3}$ & $0(0.0)$ & $5(6.8)$ & 0.587 \\
\hline CMV colitis & $0(0.0)$ & $2(2.7)$ & $>0.99$ \\
\hline \multicolumn{4}{|l|}{ GVHD } \\
\hline Acute & $1(4.5)$ & $7(9.6)$ & 0.677 \\
\hline Chronic & $1(4.5)$ & $4(5.5)$ & $>0.99$ \\
\hline \multicolumn{4}{|c|}{$\begin{array}{l}{ }^{1} \text { Other broad spectrum antibiotics: vancomycin, aztreonam. } \\
{ }^{2} \text { Other immunosuppression (prophylaxis): vorinostat, sirolimus, etanercept, extracorporeal photopheresis. } \\
{ }^{3} \text { Other immunosuppression (treatment for GVHD): alemtuzemab, cyclosporine, budesonide. } \\
\mathrm{CDI} \text {, Clostridium difficile infection; SD, standard deviation; PPI, proton pump inhibitor; H2RA, histamine-2 receptor antagonist; BL/BLI, } \beta \text {-lactam/ } \\
\text { B-lactamase inhibitor; HSCT, hematopoietic stem cell transplantation; ANC, absolute neutrophil count; GVHD, graft-versus-host disease; TNF, } \\
\text { tumor necrosis factor; CMV, cytomegalovirus. }\end{array}$} \\
\hline
\end{tabular}

Table 1 


\begin{tabular}{|c|c|c|c|}
\hline & $\begin{array}{l}\text { Recurrent } \\
(\text { rCDI }) \\
(n=22)\end{array}$ & $\begin{array}{l}\text { Non-recurrent } \\
(\mathrm{nrCDI}) \\
(n=73)\end{array}$ & $P$-value \\
\hline $\begin{array}{l}\text { Time to index CDI } \\
\text { case (median, IQR) }\end{array}$ & $3.5(0.25-6.0)$ & $7.0(2.0-33.0)$ & 0.050 \\
\hline \multicolumn{4}{|l|}{ 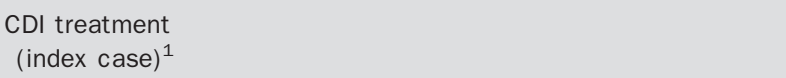 } \\
\hline Metronidazole (po) & $22(100.0)$ & 70 (95.9) & $>0.99$ \\
\hline Vancomycin (po) & $5(22.7)$ & $24(32.9)$ & 0.437 \\
\hline Combination $^{2}$ & $2(9.1)$ & $9(12.3)$ & $>0.99$ \\
\hline Other ${ }^{3}$ & $0(0.0)$ & $1(1.4)$ & $>0.99$ \\
\hline \multicolumn{4}{|c|}{$\begin{array}{l}\text { Duration of CDI } \\
\text { treatment (index case) }\end{array}$} \\
\hline$\leq 14$ days & $9(40.9)$ & $29(39.7)$ & $>0.99$ \\
\hline \multicolumn{4}{|c|}{$\begin{array}{l}{ }^{1} \text { Received at least } 1 \text { dose of the agent. } \\
{ }^{2} \text { Combination treatments: vancomycin (po) and metronidazole (IV). } \\
\text { 30ther treatments: vancomycin rectal enemas. } \\
\text { IQR, interquartile range; po, oral; IV, intravenous. }\end{array}$} \\
\hline
\end{tabular}

\section{Table 2}

disease (odds ratio [OR] 3.64, 95\% confidence interval [CI] 1.09-12.16, $P=0.040$ ) on multivariate analysis. Multiple known risk factors for recurrence in the general population appeared to predict recurrence of CDI in HSCT patients on bivariate analysis; however, these differences did not remain significant on multivariable regression analysis (Table 3 ).

In bivariate analysis in alloHSCT patients, neutropenia at the time of the index case was significantly higher in the rCDI group vs. the nrCDI group (78.8\% vs. $34.8 \%, P=0.006$ ). Among autoHSCT patients, more patients in the rCDI group had previous history of CDI (25\% vs. $0 \%, P=0.047)$. The association between neutropenia at the time of the index case and rCDI was not statistically significant in autoHSCT patients. No other variables were significantly different between the 2 groups in either transplant population.

\section{Discussion}

CDI represents a significant complication in HSCT patients. Recent studies have reported a direct correlation between early CDI and development of gastrointestinal (GI) tract GVHD with subsequent increases in mortality $(8,9)$. GVHD represents an important factor associated with overall transplant outcomes, and minimizing the development of GVHD is imperative.
Identification of risk factors for recurrence early during the transplant course may allow for utilization of preventative strategies or targeted CDI treatment during the index episode, potentially minimizing the risk of recurrent infection and HSCT-related complications.

The current literature describing $\mathrm{rCDI}$ rates in HSCT recipients or risk factors for $\mathrm{rCDI}$ in the population is limited. Previously published studies include one retrospective nested case-control study conducted by Alonso et al. (4) in autoHSCT and alloHSCT analyzing risk factors for CDI. In a subanalysis, this group found that GI GVHD conferred a nearly 5-fold higher risk for CDI recurrence after controlling for confounders (adjusted OR, 4.23 [95\% CI, 1.20-14.86]; $P=0.02$ ). This was a novel finding and further suggests a relationship between CDI and GVHD. However, this study did not account for several other well-known risk factors for rCDI including history of CDI or antibiotic use during $\mathrm{CDI}$, which may have contributed to CDI recurrence. Timing of onset of infection was also not captured.

To our knowledge, this study is the first to directly examine factors associated with rCDI in the HSCT population, including both traditional risk factors for recurrence as well as potential HSCT-related factors. We observed a $23.3 \%$ recurrence rate, which is similar to that seen in a prior study in HSCT patients (21.7\%) (5). In addition, we observed high rates of early initial CDI in our cohort irrespective of subsequent recurrence, specifically in the first 7 days post transplant.

Based on the results of our multivariate analysis, it appears that traditional risk factors for recurrence in the general hospitalized population may not be as strongly associated with rCDI in HSCT patients as other variables. Although a significant correlation was seen between recurrent infection and a prior history of $\mathrm{CDI}$ on bivariate analysis, this association became nonsignificant after accounting for other covariates. This finding may be a result of the fact that most transplant patients display many traditional risk factors for recurrence including use of broad-spectrum antibiotics and acid-suppression therapy, as part of UMHS transplant protocols.

The strongest predictor of recurrent disease for the cumulative population, based on our regression model, was neutropenia at the time of the index CDI episode. Reasons for this finding are currently not well defined. Colitis due to CDI is a neutrophilic process, and the extent to which neutrophils are required for clearance of infecting clostridia or pathophysiology of the disease is unclear. A previous case-control study in HSCT patients found that receipt of growth factor was associated with a reduced risk of rCDI (10). These 
Bivariate and multivariable unconditional logistic regression of factors present at index case that predict recurrent infection

\begin{tabular}{|c|c|c|c|c|c|c|}
\hline \multirow[b]{2}{*}{ Factors } & \multicolumn{3}{|c|}{ Bivariate analysis $^{1}$} & \multicolumn{3}{|c|}{ Multivariate logistic regression } \\
\hline & $\begin{array}{l}\text { Recurrent (rCDI) } \\
(n=22)\end{array}$ & Non-recurrent (nrCDI) $(n=73)$ & $P$-value & OR & $95 \% \mathrm{Cl}$ & $P$-value \\
\hline \multicolumn{7}{|l|}{ Traditional risk factors } \\
\hline History of CDI & $5(22.7)$ & $5(6.8)$ & 0.048 & 3.55 & $0.78-16.11$ & 0.101 \\
\hline \multicolumn{7}{|l|}{ Acid-suppression therapy } \\
\hline PPI & $9(40.9)$ & $44(60.3)$ & 0.143 & 0.40 & $0.09-1.90$ & 0.254 \\
\hline H2RA & $9(40.9)$ & $19(26.0)$ & 0.193 & 0.86 & $0.17-4.42$ & 0.855 \\
\hline \multicolumn{7}{|l|}{ Broad-spectrum antibiotics } \\
\hline Carbapenems & $2(9.1)$ & $1(1.4)$ & 0.133 & 8.13 & $0.50-133.05$ & 0.142 \\
\hline Cephalosporins & $14(63.6)$ & $32(43.8)$ & 0.144 & 1.66 & $0.54-5.13$ & 0.382 \\
\hline \multicolumn{7}{|l|}{ HSCT-related factors } \\
\hline Index case during hospitalization for HSCT & $18(81.8)$ & $48(65.8)$ & 0.192 & 1.15 & $0.27-4.85$ & 0.849 \\
\hline Neutropenic $($ ANC $<500)$ at index case & $13(59.1)$ & $21(28.8)$ & 0.012 & 3.64 & $1.09-12.16$ & 0.040 \\
\hline
\end{tabular}

Table 3

data, in addition to our findings, are consistent with neutrophils being important for the elimination of C. difficile from the GI tract, but this is an area for future research.

It is also possible that neutropenia in our study was a surrogate marker of a lack of memory cell response to past infection, or infection that occurred before engraftment. In a prospective study of 63 patients with nosocomial CDI, serial serum immunoglobulin (Ig)A, IgG, and IgM concentrations against $\mathrm{CDI}$ toxins $\mathrm{A}$ and $\mathrm{B}$ were measured and followed up for relapsed infection. Based on multivariate logistic regression analysis, the authors found that serum antibody response to toxin A during the initial episode of CDI was associated with protection against recurrence (10). In addition, a statistically significant downward trend in recurrence rates was observed with increasing serum IgM concentrations against $\mathrm{CDI}$ toxin $\mathrm{A}$, measured on day 3 of initial infection. Several other small studies analyzing the relationship between serum Ig levels and rCDI have reached similar conclusions (11-13). Thus, it could be speculated that leukopenic HSCT recipients might be unable to mount appropriate immune responses during the initial CDI episode, and may therefore be at higher risk of recurrent infection.

Similarly, disruption of the integrity of the GI mucosa by the conditioning regimen, which is most profound during the pre-engraftment period, could also play a role in recurrent infection. Studies have shown that chemotherapy-induced mucositis alters the composition of the intestinal microflora, causes reduction in epithelial repair, and impairs normal functions such as immune regulation and protection from pathogen overpopulation $(3,14,15)$. C. difficile spores may thus be more adept to survive, proliferate, and cause recurrence in patients with severely suppressed intestinal micobiota (16). A secondary finding in our study was that HSCT recipients with rCDI develop their index case significantly earlier than nrCDI patients. This correlates with our finding that neutropenia predicts recurrence and supports the association between immune deficiency and relapsed CDI.

Several limitations to this study should be noted. The retrospective nature of our study makes it difficult to account for all possible confounders that may impact recurrence. However, by performing a multivariable logistic regression, we accounted for traditional risk factors for recurrence as well as potential transplantrelated factors. Our retrospective study was also limited by our inability to accurately differentiate colonization from infection in all patients owing to inconsistencies in documentation of stool number and consistency. To minimize treatment of colonized patients, our clinical laboratory will not test solid stool for $C$. difficile and 
institutional policy recommends avoiding testing in patients with a single episode of diarrhea or for test-ofcure. In addition, our study may have been underpowered to detect a difference in rare events, such as cytomegalovirus colitis or GVHD, as a predictor of rCDI. In contrast to Alonso et al. (4), we did not find a higher rate of rCDI in patients with GI GVHD, despite having a similar sample size. The overall rate of GVHD does not appear to be higher at UMHS compared with other institutions with similar HSCT populations, thus other factors, such as differences in antimicrobial stewardship practice or infection control measures at our institution, may have impacted differences seen in incidence of rCDI. It is possible that, with a larger sample size, these factors may also predict recurrence. Finally, data for biomarkers, such as creatinine, albumin, or inflammatory markers, that have been shown to be associated with rCDI, would have been ideal to account for in our analysis; however, this information was not collected for all patients and thus was not included.

The results of our study indicate that the rate of $\mathrm{rCDI}$ in HSCT recipients is similar to that in non-HSCT hospitalized patients. However, traditional risk factors of recurrent infection do not appear to predict relapse in this population. While we did not observe a relationship between GI GVHD and recurrent disease, we saw a significant association with patients who were neutropenic at the time of the index episode of CDI, possibly related to a lack of memory response to the initial insult. In addition, we observed that patients with rCDI developed their index episode early in the posttransplant period. This finding most likely correlates with timing of neutropenia and highlights the potential need for preventative measures before or early after transplantation. Identifying risk factors for $\mathrm{rCDI}$ in the HSCT population has important implications regarding the initial management of CDI episodes. Novel treatment modalities or prevention strategies, such as pretransplant screening and decolonization, use of prophylaxis, longer duration of therapy, and pulse-dose or long-term taper antibiotic therapy, could be potential methods to minimize recurrent infection in high-risk patients. Further studies should be conducted to evaluate if targeted therapy or pre-engraftment prophylaxis should be considered in HSCT recipients with certain risk factors for recurrence.

\section{Acknowledgements:}

Support: This study was supported in part by a grant from the NIH/NIAID (5U19AI090871-02).

\section{References}

1. Willems L, Porcher R, Lafaurie M, et al. Clostridium difficile infection after allogeneic hematopoietic stem cell transplantation: incidence, risk factors, and outcome. Biol Blood Marrow Transplant 2012; 18: 1295-1301.

2. Leung S, Metzger BS, Currie BP. Incidence of Clostridium difficile infection in patients with acute leukemia and lymphoma after allogeneic hematopoietic stem cell transplantation. Infect Control Hosp Epidemiol 2010; 31: 313-315.

3. Trifilio SM, Pi J, Mehta J. Changing epidemiology of Clostridium difficile-associated disease during stem cell transplantation. Biol Blood Marrow Transplant 2013; 19 (3): 405-409. Epub 2012 Dec 4.

4. Alonso CD, Treadway SB, Hanna DB, et al. Epidemiology and outcomes of Clostridium difficile infections in hematopoietic stem cell transplant recipients. Clin Infect Dis 2012; 54 (8): 1053-1063.

5. Eyre DW, Walker AS, Wyllie D, et al. Predictors of first recurrence of Clostridium difficile infection: implications for initial management. Clin Infect Dis 2012; 55 (S2): S77-S787.

6. McDonald LC, Coignard B, Dubberke E, Song X, Horan T, Kutty PK. Recommendations for surveillance of Clostridium difficileassociated disease. Infect Control Hosp Epidemiol 2007; 28: 140-145.

7. Glucksberg H, Storb R, Fefer A, et al. Clinical manifestations of graft-versus-host disease in human recipients of marrow from HL-A-matched sibling donors. Transplantation 1974; 18: 295-304.

8. Chakrabarti S, Lees A, Jones SG, Milligan DW. Clostridium difficile infection in allogeneic stem cell transplant recipients is associated with severe graft-versus-host disease and non-relapsemortality. Bone Marrow Transplant 2000; 26 (8): 871-876.

9. Dubberke ER, Reske KA, Srivastava A, et al. Clostridium difficileassociated disease in allogeneic hematopoietic stem-cell transplant recipients: risk associations, protective associations, and outcomes. Clin Transplant 2010; 24: 192-198.

10. Kyne L, Warny M, Qamar A, Kelly CP. Association between antibody response to toxin $\mathrm{A}$ and protection against recurrent Clostridium difficile diarrhoea. Lancet 2001; 357: 189-193.

11. Leung DY, Kelly CP, Boguniewics M, Pothoulakis C, LaMont JT, Flores A. Treatment with intravenously administered gamma globulin of chronic relapsing colitis induced by Clostridium difficile toxin. J Pediatr 1991; 18: 633-637.

12. Warny M, Vaerman JP, Avesani V, Delmee M. Human antibody response to Clostridium difficile toxin A in relation to clinical course of infection. Infect Immun 1994; 62: 384-389.

13. Bacon AE 3rd, Fekety R. Immunoglobulin G directed against toxins A and B of Clostridium difficile in the general population and patients with antibiotic-associated diarrhea. Diagn Microbiol Infect Dis 1994; 18: 205-209.

14. Stringer AM, Gibson RJ, Bowen JM, Keefe DMK. Chemotherapy-induced modifications to gastrointestinal microflora: evidence and implications of change. Curr Drug Metab 2009; 10 (1): 79-83.

15. Bodet CA 3rd, Jorgensen JH, Drutz DJ. Antibacterial activities of antineoplastic agents. Antimicrob Agents Chemother 1985; 28 (3): 437-439.

16. Babakhani F, Bouillaut L, Gomez A, Sears P, Nguyen L, Soneshein AL. Fidaxomicin inhibits spore production in Clostridium difficile. Clin Infect Dis 2012; 55 (S2): S162-S169. 